\title{
Philosophiques
}

\section{Agir sur les coeurs : spectacle et duplicité chez Rousseau}

\section{Philip Knee}

Volume 14, numéro 2, automne 1987

URI : https://id.erudit.org/iderudit/027015ar

DOI : https://doi.org/10.7202/027015ar

Aller au sommaire du numéro

Éditeur(s)

Société de philosophie du Québec

ISSN

0316-2923 (imprimé)

1492-1391 (numérique)

Découvrir la revue

Citer cet article

Knee, P. (1987). Agir sur les coeurs : spectacle et duplicité chez Rousseau. Philosophiques, 14(2), 229-327. https://doi.org/10.7202/027015ar

\section{Résumé de l'article}

Le caractère complexe de la notion d'« opinion » chez Rousseau, qui est à la fois une soumission à l'estime des autres et un levier indispensable pour toute réforme des moeurs, est la base d'une discussion des enjeux de l'action qu'il prône sur les coeurs des hommes : d'abord par l'évocation de quelques thèmes de la Lettre à d'Alembert qui tente de définir les difficultés de cet " art "; ensuite au niveau de l'action positive des grands réformateurs : le Législateur, le gouverneur d'Emile et surtout Wolmar dont l'échec dans La Nouvelle Hélö̈se jette un éclairage décisif sur l'ensemble de la démarche éthique de Rousseau.
Ce document est protégé par la loi sur le droit d'auteur. L'utilisation des services d'Érudit (y compris la reproduction) est assujettie à sa politique d'utilisation que vous pouvez consulter en ligne.

https://apropos.erudit.org/fr/usagers/politique-dutilisation/ 


\title{
AGIR SUR LES COEURS : SPECTACLE ET DUPLICITÉ CHEZ ROUSSEAU
}

\author{
par Philip Knee
}

\begin{abstract}
RÉsumÉ. Le caractère complexe de la notion d' «opinion》 chez Rousseau, qui est à la fois une soumission à l'estime des autres et un levier indispensable pour toute réforme des mœurs, est la base $d$ 'une discussion des enjeux de l'action qu'il prône sur les cœurs des hommes: d'abord par l'évocation de quelques thèmes de la Lettre à d'Alembert qui tente de définir les difficultés de cet «art »; ensuite au niveau de l'action positive des grands réformateurs : le Législateur, le gouverneur d'Émile et surtout Wolmar dont l'échec dans La Nouvelle Héloïse jette un éclairage décisif sur l'ensemble de la démarche éthique de Rousseau.
\end{abstract}

\begin{abstract}
The complex character of "opinion" in the works of Rousseau, which is both a submission to the judgment of others and an indispensable leverage for the reform of morals, is the basis for a discussion of his action on the hearts of men : first through some of the themes of the Lettre à d'Alembert which attempts to define the difficulties of such an "art"; then through the action of the great reformers : the Legislator, Emile's governor and especially Wolmar, whose failure in La Nouvelle Heloïse sheds an important light on the whole of Rousseau's ethical endeavour.
\end{abstract}

À l'origine des nombreux paradoxes que soulève la pensée de Rousseau, on peut parler d'une figure de redoublement où ce qui semble condamné d'une part comme la source du dérèglement de l'existence est repris et réaffirmé d'autre part comme la voie de sa réforme possible. On sait qu'à partir de la multiplication des besoins et des rapports des hommes, Rousseau diagnostique leur corruption dans le paraître, dans l'oubli de soi, dans le tumulte du monde, et qu'il en appelle à un travail de l'homme sur lui-même 
pour " se circonscrire " ${ }^{1}$, se défaire des masques et des artifices de la vie sociale. C'est une éthique de la transparence qui est ainsi visée, atteignable par la fidélité de chacun à la voix de la nature en lui contre les sollicitations de l'extérieur qui arrachent l'homme à lui-même. Mais il s'agit justement d'un travail, non d'un pur élan de spontanéité. Dans ce sens, se défaire du paraître signifie, au niveau individuel, la conquête d'une solitude qui n'est nullement donnée, et cela par un effort de maîtrise de soi et de ses passions qui implique justement de se masquer, sans quoi l'on s'interdit toute prise en charge de ses responsabilités vis-à-vis des autres. Au niveau politique, pareillement, ce n'est que par les artifices et une dénaturation exacerbée qu'il semble possible de rejoindre par l'ordre social adéquat les valeurs de l'existence naturelle. Tout en s'opposant au paraître, Rousseau reconnaît donc que celui-ci est omniprésent, qu'il faut en passer par lui, et si cette dénonciation semble permettre d'asseoir une éthique et de rendre intelligibles les rapports entre les hommes, la dénonciation ne saurait d'ellemême constituer cette éthique. Celle-ci requiert un effort autrement plus complexe et patient : d'utiliser contre le mal les armes du mal lui-même ${ }^{2}$.

Cette figure révèle avec force ses richesses et ses difficultés lorsque Rousseau aborde - comme il ne cesse de le faire d'un bout à l'autre de son cuvre - la question de savoir comment façonner les mours des hommes ${ }^{3}$, c'est-à-dire lorsqu'il prétend se tenir sur cette crête difficile où il utilise l'amour-propre des hommes, leur sujétion à l'opinion, pour les rendre libres. Terme ambigu et jamais défini chez Rousseau, l'opinion désigne d'abord ce qui en l'homme n'est pas de l'ordre de la nature, ce qui lui vient de l'extérieur et par là altère la voix de sa conscience. Le «poison de l'opinion ${ }^{4}$ est ce qui existe en lui seulement par l'estime des

1. Les Rêveries du promeneur solitaire, Cinquième promenade, Euvres complètes de Rousseau dans l'édition de la Pléiade (PI.), tome I, p. 1040; voir aussi les Lettres morales, PI. IV, p. 1112.

2. Voir Du Contrat social (première version), Pl. III, p. 288; ec Préface à Narcisse ou l'amant de lui-même, Pl. II, p. 972. Soulignons que J. STAROBINSKI a fort bien mis en évidence cette figure chez Rousseau, en particulier pour la question de la réflexion : “J. J. Rousseau ou le péril de la réflexion" dans L'oeil vivant, Gallimard, 1961.

3. C'est le thème privilégié par Rousseau quand il tente de s'expliquer sur son œuvre dans la Lettre à C. de Beaumont, Pl. IV, pp. 927-1007.

4. Émile ou de l'éducation, Pl. IV, p. 444. 
autres et nie donc son indépendance originelle, faisant de lui un être dépendant ${ }^{5}$, affaibli, aliéné. Mais seul Dieu, pour Rousseau, est pleinement indépendant, et cette dépendance de l'homme est donc tributaire d'une faculté fondamentalement humaine, celle de s'objectiver : la conscience de soi qui naît du regard porté sur soi-même à partir du regard de l'autre ${ }^{6}$. Ainsi se trouve relativisée toute critique de l'opinion, en étant rapportée à un caractère spécifiquement humain que la morale doit assumer comme constitutive de sa propre démarche. Au niveau collectif, l'opinion désigne, dans le même sens, les préjugés communs d'un groupe, les croyances et les normes non réfléchies de la conscience collective 7 , ou plutôt, pourrait-on dire, de son inconscience. Mais il s'agit en même temps de ce qui fait qu'une communauté d'hommes existe comme telle, c'est-à-dire communique et règle ses conflits autrement que par la force. Comme représentation d'elle-même à travers des structures symboliques, l'opinion est la condition d'existence d'une culture, condition que toute pensée politique doit d'abord reconnaître pour se constituer et envisager une action.

On comprend alors en quel sens Rousseau va privilégier l'action sur les hommes par l'opinion elle-même, puisque c'est en prenant en charge le paraître qui est constitutif de son être dénaturé que l'existence sociale peut espérer se réformer. Par ce biais de l'image que les hommes ont toujours d'eux-mêmes par les autres, et en prenant pour modèle l'autorité naturelle du père de famille, la règle politique sans cesse réitérée par Rousseau est d'agir sur les hommes par le dedans, au niveau de leurs désirs et de leurs espoirs, plutôt que de compter sur le raisonnement ou la contrainte. «L'autorité la plus absolue, dit-il, est celle qui pénètre jusqu'à l'intérieur de l'homme, et ne s'exerce pas moins sur la volonté que sur les actions ${ }^{8}$. Si l'on réussit à agir sur les cœurs, pas besoin de contrainte ni même de persuasion: les hommes

5. Dans un utile article, $\mathrm{L}$. CROCKer rassemble à travers ce thème de la dépendance les différentes utilisations du mot par Rousseau : Rousseau et l'opinion, dans Studies on Voltaire and the Eighteenth century, $n^{\circ} 55,1967$.

6. Voir le Discours sur l'origine et les fondements de l'inégalité parmi les hommes (Deuxième Discours), PI. III, PP. 164-70.

7. C. GANOCHAUD passe en revue toutes les manifestations de ce thème: L'opinion publique chez J.J. Rousseau, Atelier de reproduction des thèses de l'Université de Lille III, 1980.

8. Discours sur l'économie politique, PI. III, p. 251. 
seront justes et bons sans même savoir ce qu'est la justice et la bonté ${ }^{9}$. Mais cette règle est fonction des sociétés, des groupes, des individus auxquels on a affaire, c'est-à-dire de «l'état de l'opinion » en chaque situation singulière et, partant, de ce qu'il semble possible d'en espérer. C'est sur ce travail de diagnostic et ce qui en découle chez Rousseau, que nous voudrions attirer l'attention ici, en nous appuyant d'abord sur sa Lettre à d'Alembert de 1758 , texte stratégique situé (théoriquement sinon chronologiquement) à l'articulation des Discours et des grandes œuvres réformatrices, et dont les considérations négatives sur le problème du spectacle dans la société n'ont peut-être pas été suffisamment mises à contribution par les commentateurs ${ }^{10}$; en évoquant ensuite le terrain beaucoup plus connu des réponses positives apportées par Rousseau, et particulièrement celle qu'incarne l'action de Wolmar dans La Nouvelle Héloïse.

\section{Les apparences}

Il s'agit, comme l'on sait, d'un débat sur l'introduction du théâtre à Genève, idée prônée par d'Alembert dans son article de l'Encyclopédie consacré à la ville d'origine de Rousseau et à laquelle celui-ci répond longuement pour s'y opposer. Mais le théâtre n'est pour Rousseau qu'une occasion particulièrement propice pour réfléchir sur le problème moral et politique mis en jeu par l'article : celui du rapport entre les mœurs d'une communauté et la représentation de celles-ci, entre la vie d'un peuple et le spectacle qu'on en donne, car là se trouvent concentrés tous les problèmes de cette action sur les cœurs évoquée plus haut. $\grave{A}$ ce titre, il importe de marquer d'abord la pertinence de la controverse sur l'autorité religieuse dont les premières pages de la Lettre se font l'écho ${ }^{11}$, car sous une forme rudimentaire, c'est la question de fond qui nous occupe ici qui s'y trouve indiquée.

9. Voir Projet de Constitution pour la Corse, PI. III, p. 948.

10. Notons toutefois le récent essai de P. COLEMAN, Rousseau's political imagination, Rule and representation in the Lettre d̀ d'Alembert, Droz, 1984, qui propose une lecture soigneuse du texte.

11. Nous renvoyons à l'édition Garnier-Flammarion, 1967, de la Lettre à d'Alembert sur son article «Genève» (Lettre), pp. 55-63. 
Dans le cadre de son éloge de Genève, d'Alembert loue les pasteurs calvinistes de cette ville et leur " socinianisme parfait» ${ }^{12}$. Le contexte permet de situer cette remarque et la réaction de Rousseau. Récemment installé près de Genève, c'est Voltaire qui sollicite les talents de d'Alembert afin de favoriser, à travers cet article espère-t-il, ses plans de voir s'installer dans la ville un théâtre où il fera représenter ses cuvres. L'article s'inscrit donc dans le cadre d'un projet politique particulier et dans celui de l'offensive générale des «Philosophes» en faveur de la librepensée, contre la religion et en particulier contre l'intolérance et le dogmatisme ${ }^{13}$. C'est ainsi que Voltaire et d'Alembert semblent s'être laissés abuser par les apparences anti-dogmatiques de quelques pasteurs genevois surtout soucieux de politesse, et avoir été trop contents de ne retenir de leurs contacts avec eux que leur anticatholicisme, pour ne les voir que comme des sceptiques du dogme et des champions de la tolérance ${ }^{14}$. D'où cette étiquette " socinienne » qui fit scandale. L'on jugea que d'Alembert s'était servi des apparences pour faire avancer la cause de l'Encyclopédie et qu'un tel éloge venant d'une telle source ne pouvait que porter préjudice à l'autorité de l'Église genevoise. Le débat confus qui se poursuit à ce sujet dans les premiers mois de 1758 (demande de rétractation, à laquelle d'Alembert se dérobe, etc.) provoque ces pages de la Lettre de Rousseau, où il se range fermement du côté des pasteurs mais où est visé un enjeu politique beaucoup plus général.

12. Lettre, p. 57 ; voir d'AlemberT, Article "Genève » dans l'Encyclopédie ou Dictionnaire raisonné des Sciences, des Arts et des Métiers (extraits), Garnier-Flammarion, 1986, vol. 2, p. 141.

Considéré comme un « libéralisme » protestant et répandu dans la plupart des régions atteintes par la Réforme, le socinianisme issu de l'enseignement de Fausto Socin se caractérise par la négation de la prédestination et de l'inspiration totale des Écritures, la liberté d'examen, la conception symbolique des sacrements, etc. (d'après le Grand Larousse Encyclopédique). Voir à ce sujet: Voltaire, Lettres pbilosophiques, VII, Gallimard, 1986.

13. Voltaire se vante de vouloir «pervertir cette cité pédante qui conservait un bon souvenir de ses réformateurs (...) et croyait à la parole de ses prédicants»: cité dans M. Moliat, Rousseat et la querelle du théatre au XVIII siècle (1930), Slatkine Reprints, Genève, 1970, p. 62.

14. Voir l'explication de d'Alembert à la fin de la réponse qu'il fera à la Lettre de Rousseau: Lettre à M. Rousseau dans Mélanges de littérature, d'bistoire et de philosophie, Zacharie-Chatelain et fils, Amsterdam, 1764-1767, Tome deuxième, pp. 449-53. Dans le Tome cinquième du même ouvrage, d'Alembert rassemble plusieurs éléments du dossier, dont une « justification » de l'article de l'Encyclopédie, un extrait de la déclaration des pasteurs qu'il provoqua (février 1758), et un extrait de la Lettre de Rousseau (mars 1758). 
C'est le sujet, dit Rousseau, «dont l'examen me convient le moins " ${ }^{15}$, mais s'il s'y consacre dès le début de son texte sur les spectacles, c'est bien qu'il met en place — serait-ce discrètement le problème des apparences politiques, celui des liens entre les croyances personnelles et leur expression dans la cité, des liens entre l'autorité publique (en l'occurrence l'Église) et les mœurs d'une communauté. En effet, Rousseau ne discute pas le dogme. Il dit sentir «plus d'éloignement que de goût» pour la secte socinienne ${ }^{16}$ et ne pas même connaître sa doctrine. Ce qui lui importe n'est pas de savoir si les pasteurs y adhèrent, même s'il reproche à d'Alembert de ne pas le savoir assez directement ; c'est le fait que celui-ci se soit cru permis de les désigner ainsi publiquement, de faire un éloge qui les mette en difficulté. Le reproche en est un de forme essentiellement : «Mais Monsieur quand on veut honorer les gens, il faut que ce soit à leur manière, et non à la nôtre; de peur qu'ils ne s'offensent avec raison des louanges nuisibles qui, pour être données à bonne intention, n'en blessent pas moins l'état, l'intérêt, les opinions ou les préjugés de ceux qui en font l'objet. (...) Cette prétendue vérité n'est pas si claire ni si indifférente que vous soyez en droit de l'avancer sans de bonnes autorités ${ }^{17}$. Une vérité bonne à dire dépend de celui à qui l'on s'adresse et dans quel but, suggère Rousseau, anticipant sa fameuse discussion sur la vérité et la véracité de la quatrième promenade des Rêveries. La vérité est un problème pratique et, dans ce cas, politique, et il peut y avoir des duplicités nécessaires qu'il faut savoir comprendre et respecter, ce dont semblent incapables d'Alembert comme d'ailleurs, aux yeux de Rousseau, tous les "Philosophes", sur les questions religieuses et politiques.

Ce qui rend ces pages en apparence énigmatiques, c'est que Rousseau y témoigne simultanément de deux préoccupations qui, en surface, semblent s'opposer, et sur lesquelles, au fil des notes surtout, il ne lâche pas prise. D'un côté, s'il n'est pas «socinien » lui-même, il n'est pourtant pas éloigné des positions éclairées de cette secte et donc de l'intention de d'Alembert lorsque celui-ci fait l'éloge de son ouverture d'esprit. Rousseau dira plus tard que l'Église genevoise lui avait semblé plus proche de l'esprit véritable

15. Lettre, p. 55.

16. Lettre, p. 58.

17. Lettre, p. 56. 
du christianisme ${ }^{18}$ par l'importance accordée à la conscience individuelle et à sa liberté de jugement en matière de dogme. Le Vicaire savoyard de Rousseau, on le sait, recule devant certaines contradictions du dogme et prend le chemin de la religion naturelle - et c'est évidemment cet aboutissement logique de la mise en cause du dogme qui faisait tant craindre le socinianisme. Il est vrai que pour le socinien il s'agit «de ne rien proposer à croire qui heurte la raison ", comme le dit d'Alembert, ce qui n'est pas vraiment la position du Vicaire, qui insiste sur les limites de l'entendement humain, l'humilité intellectuelle nécessaire et le doute respectueux en matière de croyance. Cela dit, voulant s'opposer d'abord aux fanatismes, Rousseau conclut surtout qu'il faut laisser le socinien «en repos». Mais, de l'autre côté, l’on a vu que ce qui préoccupe centralement Rousseau dans la Lettre et provoque sa réaction, c'est l'affaiblissement corrélatif de l'autorité des pasteurs à Genève, ce qui fait qu'indépendamment du débat religieux, il veut les défendre contre l'étiquette socinienne donnée avec une légèreté coupable (ou perverse) par d'Alembert. Tout en prônant le rôle de la conscience de chacun en matière religieuse, Rousseau dit vouloir exposer "des vérités de pratique » afin de «remplir son devoir» en s'adressant «au plus grand nombre» ${ }^{19}$. On voit donc que ce sont deux exigences politiques générales qui s'affrontent par la voix de Rousseau sur une question religieuse particulière: il nous laisse entrevoir le paradoxe de vouloir penser en même temps l'exigence de la liberté de conscience de l'individu, de la critique légitime des institutions sociales et des apparences; et

18. ROUSSEAU revient non sans amertume sur la controverse quelques années plus tard à l'occasion d'une autre polémique concernant Genève, dans ses Lettres écrites de la montagne, Pl. III, PP. 717-8: "L'Église de Genève paraissait depuis longtemps s'écarter moins que les autres du véritable esprit du Christianisme et c'est sur certe trompeuse apparence que $j$ 'honorai ses Pasteurs d'éloges dont je les croyais dignes ; car mon intention n'était assurément pas d'abuser le public (...). Un Philosophe jette sur eux un coup d'œil rapide; il les pénètre, il les voit Ariens, Sociniens; il le dit et pense leur faire honneur : mais il ne voit pas qu'il expose leur intérêt temporel ; la seule chose qui généralement décide ici-bas de la foi des hommes. Aussitôt alarmés, effrayés, ils s'assemblent, ils discutent, ils s'agitent, ils ne savent à quel saint se vouer; et après force consultations, délibérations, conférences, le tout aboutit à un amfigouri où l'on ne dit ni oui ni non, et auquel il est aussi peu possible de rien comprendre qu'aux deux plaidoyers de Rabelais». On voit que Rousseau se révèle plus proche ici des attaques des Encyclopédistes sur l'hypocrisie de l'Église, puisqu'il s'agit davantage de dénoncer la duplicité des pasteurs que de défendre leur autorité comme dans la Lettre.

19. Voir les deux notes qui se suivent dans la Lettre, pp. 58-60. 
l'exigence de l'identification à l'autorité, nécessaire à la vie dans la cité comme cette part d'inconscience requise par tout ordre social ${ }^{20}$. De plus, en soulignant simultanément l'inconvenance politique et l'importance morale de la caractérisation des pasteurs par d'Alembert, Rousseau met son lecteur au fait de la difficulté de son propre texte alors qu'il va aborder le problème du théâtre. C'est dans cette perspective qu'il faut envisager la forme de la Lettre, ses constantes digressions qui ne semblent jamais affronter la substance des problèmes, le ton soudain très personnel de certains passages, l'oscillation entre l'essai théorique et la confidence. Cette forme bâtarde témoigne justement des paradoxes impliqués dans l'intervention dans les affaires publiques c'est-à-dire dans la manière dont les hommes se représentent ce qu'ils vivent : à sa façon, la Lettre participe de ce qu'on doit appeler une stratégie du paraître.

\section{Le théâtre}

Ces paradoxes étaient déjà d'une certaine manière ceux du Discours surles Sciences et les Arts ${ }^{21}$. On sait que sur le conseil de Diderot à Vincennes, Rousseau avait choisi dans ce texte le parti de l'originalité dans sa réponse à la question de l'Académie de Dijon en se plaçant à contre-courant des idées de l'époque, parti qui lui avait valu une certaine célébrité mais aussi la réputation d'être un écrivain aux paradoxes sans doute brillants mais peu sérieux. Dans sa préface à Narcisse, en 1753, Rousseau revenait sur ceux-ci pour préciser sa condamnation des sciences et des arts : la corruption des mours des grandes villes a produit des besoins qui, aussi factices qu'ils soient, sont si enracinés qu'il peut être inutile et même dangereux de vouloir s'y opposer ${ }^{22}$. Mais l'a-t-on bien compris? Le débat sur le théâtre à Genève - petite communauté encore peu corrompue où les divertissements des grandes villes auraient justement un effet dévastateur ${ }^{23}$ — est l'occasion de

20. Sur ce thème général du rapport du savoir et de la société chez Rousseau, voir la brillante mise en place des termes du problème par L. STRauss, dans Droit naturel et Histoire, Plon, 1954, ch. VI.

21. Notons que c'est d'emblée au Premier Discours que revient d'Alembert dans sa réponse à Rousseau, pour comprendre le sens de la Lettre: Lettre à M. Rousseau, op. cit.

22. Préface à Narcisse ou l'amant de lui-même, Pl. II, pp. 971-3.

23. C'est la thèse centrale que Rousseau oppose à d'Alembert dans la Lettre, en l'appuyant sur l'évocation très personnelle de la vie des Montagnons du Valais : pp. 130-143. 
s'expliquer à nouveau, d'illustrer les thèses du Premier Discours et de se justifier à travers une situation qui engage directement Rousseau par ses idées et ses origines. Nouvellement reconverti au protestantisme, il est sur le point de retourner s'installer dans sa ville quand Voltaire commence à y faire sentir son influence : «Dès lors je tins Genève perdue et je ne me trompai pas », dira-t-il plus tard ${ }^{24}$, allant jusqu'à soupçonner Voltaire d'être l'auteur caché du paragraphe sur le théâtre dans l'article de d'Alembert alors qu'il semble n'en avoir été que l'inspirateur. C'est de là et non de Rousseau lui-même que vient la place de ce thème dans le débat avec l'Encyclopédie, puisque l'éloge qui est fait de Genève n'est terni, artificiellement, que de ce seul point noir: l'absence de comédie; et Rousseau flaire évidemment la conspiration lorsque d'Alembert déclare voir dans l'introduction du théâtre le moyen «de réformer l'Europe sur ce point » en faisant des bonnes mours de Genève un «modèle » pour le théâtre des autres nations ${ }^{25}$. De fait, dans la querelle sur le théâtre qui bat son plein au XVIIIe siècle après les sévères condamnations de Bossuet, Voltaire a pris ardemment parti pour le théâtre comme instrument de civilisation et contre les préjugés qui affligent les acteurs ; et dans son sillage Diderot s'est fait l'apôtre du rôle moral et politique du théâtre dans la société. C'est dans ce climat philosophique dont la querelle théâtrale n'est que la pointe visible mais combien exemplaire, ainsi que dans l'attachement de Rousseau pour Genève, dont il évoquera plus tard la force non sans un peu d'ironie ${ }^{26}$, qu'il faut voir l'origine de l'engagement personnel dont témoigne la Lettre ${ }^{27}$, plutôt, nous semble-t-il, que dans l'activité théâtrale de Rousseau lui-même et l'importance psychologique de ses échecs d'auteur et

24. Les Confessions, Pl. I, p. 396.

25. Voir la citation du paragraphe de d'Alembert dans la Lettre, p. 46; et dans l'Encyclopédie, op. cit., p. 140.

26. «Jamais je n'ai vu les murs de cette heureuse ville, jamais je n'y suis entré sans sentir une certaine défaillance de cœeur qui venait d'un excès d'attendrissement. En même temps que la noble image de la liberté minélevait l'âme, celles de l'égalité, de l'union, de la douceur des mœurs me touchaient jusquaux larmes et m'inspiraient un vif regret d'avoir perdu tous ces biens. Dans quelle erreur j'étais, mais qu'elle était naturelle! Je croyais voir tout cela dans ma patrie parce que je le portais dans mon coeur » : Les Confessions, Pl. I, p. 144.

27. Voir Les Confessions, PI. I, pp. 494-6. Pendant la rédaction de la Lettre, Rousseau est persuadé qu'il n'écrira plus, que son œuvre est terminée, alors que s'ouvre en fait sa grande période dę solitude créatrice entre 1757 et 1762 . 
d'acteur avant la conversion de Vincennes ${ }^{28}$. Il n'empêche que c'est bien le comédien qui sera l'adversaire privilégié dans la Lettre, Rousseau rejoignant ainsi les attaques traditionnelles de l'Église sur l'immoralité des acteurs même si le sens de sa critique n'a rien de chrétien : il n'argumente qu'en fonction du corps social non de la foi et entend se situer du côté du peuple non des prêtres. Mais la nouveauté de sa position et ce qui lui donne tant d'écho, c'est justement qu'elle reprend d'un point de vue laïc la thèse catholique et la renouvelle en l'appliquant à un cas particulier ${ }^{29}$.

Rousseau s'attaque donc à la conception de l'art du comédien qui est dominante dans les textes des Encyclopédistes ${ }^{30}$, mais dont la meilleure expression, et celle que retiendra la postérité, se trouve dix ans après la Lettre sous la plume de Diderot. Dans son Paradoxe sur le comédien ${ }^{31}$, le problème technique discuté est celui des meilleurs moyens pour le comédien de «faire vrai», de faire « passer » l'illusion sur scène : identification réelle de l'acteur avec le personnage ou simulation adroite par une distance maîtrisée ? On sait que Diderot prône la solution extrême de l'absence de sensibilité de l'acteur; mais, plus généralement, on insiste à l'époque (tout comme ceux qui attaquaient les acteurs, d'ailleurs, pour fonder leur condamnation) sur la nécessité pour l'acteur de se métamorphoser, de cesser d'être soi, de se déprendre de lui-même pour jouer le personnage - le caractère propre du grand acteur étant de n'en avoir aucun, selon la formule à l'origine du texte de Diderot. Cette description favorable devient chez Rousseau l'occasion d'une critique des masques de l'homme social moderne dont l'acteur, par ses métamorphoses successives, représente l'idéal. Dupe de lui-même, il est l'artisan de sa propre dépersonnalisation et devient même impropre, dit sévèrement Rousseau, au personnage d'homme. Mettant publiquement sa personne en vente, il «se

28. Dans son récent ouvrage, $A$. Philonenko insiste sur l'échec de Rousseau l'acteur en 1748 dans L'engagement téméraire, et sur celui de l'auteur dramatique avec ses sept pièces qui ne forment qu'un tissu de «banalités »; et il voit là un " ferment psychologique " pour l'attaque de la Lettre sur le théâtre, et même pour la critique générale du paraître amorcée dans le Deuxième Discours: Jean-Jacques Rousseau ou la pensée du malbeur, Vrin, 1984, Vol. I, Le traité du mal, Ch. II.

29. Voir à ce sujet, M. MOFFAT, op. cit., p. 97.

30. Sur les auteurs concernés, voir J. RoussET, «Qu'est-ce que le talent du comédien? 》, dans Annales de la Société J. J. Rousseau, $\mathrm{n}^{\circ} 37,1966-8$.

31. Ce dialogue est rédigé par DiDerot à partir de 1769; voir Euvres, Édition de la Pléiade, 1971. 
soumet aux affronts qu'on achète le droit de lui faire " $^{32}$; et Rousseau révèle l'ambition de sa critique en rapprochant les «Philosophes » de cette posture publique de l'acteur dont le public attend qu'il soit autre que lui-même, et en la contrastant avec celle de l'orateur, citoyen remplissant sa fonction ${ }^{33}$. C'est la scission de l'être et du paraître, inhérente à la vie sociale analysée dans le Deuxième Discours, que Rousseau voit s'incarner dans cet anéantissement de soi, cet art de la fausseté qui se plaît à représenter aux hommes un mensonge vivant pour le faire admirer. L'art du comédien, non seulement reflète, mais conforte et accentue le paraître social. La société est déjà théâtrale, et le théâtre, comme miroir trompeur de la société ${ }^{34}$, invite les hommes à s'y complaire : déjà dépravés, ils le seront une fois encore.

Ce redoublement est essentiel dans la critique de Rousseau, car face aux Encyclopédistes tout se joue autour du potentiel de regénération morale des mœurs par le théâtre. Ceux qui y voient une école de la vertu s'inspirent de la catharsis aristotélicienne pour justifier la tragédie: le spectacle des passions déréglées «purge» le spectateur ${ }^{35}$ et peut stimuler chez lui les passions opposées, le protégeant des excès; et pour la comédie, c'est le ridicule représenté qui peut corriger les travers des hommes. Mais pour Rousseau, ce potentiel correcteur du théâtre est nul, et même il accentue le mal, du moins en ce qui concerne les spectacles modernes tels qu'on entend les importer de Paris à Genève. Ce théâtre ne purge que les passions qu'on n'a pas, tout en fomentant celles qu'on a ${ }^{36}$, et puisque tout ce qui est bon en l'homme est déjà en lui, le théâtre n'est capable que de créer des émotions superficielles et ne peut provoquer de lui-même des sentiments vertueux ${ }^{37}$. Rejoignant la méfiance de Platon dans La République, Rousseau juge que la tragédie comme la comédie disposent l'âme à des

32. Lettre, p. 163.

33. Lettre, p. 164.

34. Voir ce thème dans la lettre de Saint-Preux à Julie sur les spectacles parisiens, qui est contemporaine de la Lettre: Julie ou la Nouvelle Héloïse (N. H.), Pl. II, 2e partie, XVII.

35. Lettre, p. 71.

36. Lettre, p. 74

37. La pitié, par exemple, est un sentiment virtuel mais naturel à l'homme, comme le montrait le Deuxième Discours ; et le théâtre ne peut donc que le «faire ressentir » momentanément : Lettre, p. 78. 
sentiments trop tendres qu'on satisfait ensuite aux dépens de la vertu, que loin d'être ainsi éloignée des passions nocives, notre âme est nourrie et flattée malgré elle à force de les voir représentées ; et il insiste tout particulièrement sur l'amour et sur toutes ces situations où s'étale la domination des jeunes sur les plus vieux et des femmes sur les hommes. La raison, enfin, qui seule pourrait s'opposer aux passions, n'a pas sa place sur la scène où elle ne produit que l'ennui ${ }^{38}$. S'il veut être représenté, en effet, un auteur n'a d'autre alternative que de " suivre » et d' «embellir » l'opinion ${ }^{39}$, s'interdisant alors de heurter le goût du public et donc d'agir sur ses mœurs.

La condamnation semble sans équivoque; mais souvenonsnous que ce n'est pas le principe de tout spectacle qui est rejeté, mais son sens à Genève et les formes qu'il prend dans le théâtre moderne ${ }^{40}$. C'est en ces circonstances que Rousseau se fait platonicien et déploie les arguments classiques : la raison n'y a pas sa place, les passions y sont exacerbées, l'opinion y est confortée, car les spectacles ne sont que des imitations des apparences. La vie sociale n'étant qu'ombres projetées, les spectacles permettent à un public avide d'ombres de s'y complaire; er ce qui provoque tant Rousseau, c'est que se sont les «Philosophes » qui s'en font les hérauts les plus éloquents. Cela dit, on peut imaginer que certains spectacles puissent agir positivement sur les mours s'ils tirent leur inspiration, non de l'opinion, mais d'une connaissance de la nature de l'homme qui l'incite à la vertu et lui fasse voir ce qui est en lui, à condition de rendre cet univers palpable par l'«art » approprié, c'est-à-dire par les médiations dramatiques nécessaires ${ }^{41}$. On pourrait à partir de là s'interroger sur le sens des «spectacles» de l'œuvre même de Rousseau, avant tout La Nouvelle Hélö̈se, en chantier au moment de la rédaction de la Lettre. Mais contentons-nous d'identifier la question sur laquelle débouche, dans la Lettre, l'analyse du théâtre.

38. Lettre, p. 69 et 73 .

39. Corneille et Molière n'ont rien fait d'autre, dit Rousseau, que suivre et développer le goût du public: Lettre, pp. 69-70.

40. Ce qui nous importe ici c'est avant tout cette intention philosophique de Rousseau, non ses arguments sur le théâtre eux-mêrnes qui souvent nous laissent fort insatisfaits ; d'Alembert n'a d'ailleurs aucun mal à en faire une critique solide et parfois ironique dans sa réponse à Rousseau, op. cit.

41. Rousseau donne l'exemple de la pièce de Voltaire, Nanine (Lettre, p. 76, note), vertueuse mais mal reçue par son manque d'art; ce qui fait de Voltaire - adversaire véritable de la Lettre - l'auteur dramatique qui y est le plus loué ! 
On aura peut-être remarqué, en effet, la difficulté sur laquelle repose cette analyse: le théâtre est inutile, dit en substance Rousseau, parce qu'il ne peut agir sur les mœurs, et il échoue tout particulièrement au niveau de l'opinion des hommes ${ }^{42}$ car il en tire son contenu et la condition de son succès; mais pour cette raison même, semble dire aussi Rousseau, le théâtre est nocif en flattant les passions des hommes et en accentuant par là leurs mœurs dépravées. La condamnation repose donc contradictoirement sur l'effet sur les mœurs et sur l'incapacité d'agir sur elles; sur le fait de mimer l'opinion et de la façonner. Le théâtre semble incarner concrètement le paradoxe d'une opinion qui reproduit passivement les mours des hommes $e t$ qui possède un empire sur elles. Il y a là, pour Rousseau, un enjeu directement politique, et tout son effort (comme on l'a vu avec le problème des pasteurs genevois) consiste alors à « travailler » ce paradoxe et à en infecter ses lecteurs pour leur en faire apercevoir la portée.

D'où l'interrogation qui constitue le pivot de la Lettre 43 : les lois comme la raison sont impuissantes, car ce qui gouverne les hommes c'est l'opinion dont même les rois sont esclaves; et c'est donc sur elle qu'il faut agir. Saura-t-on en trouver l'«art » ${ }^{44}$ ? Mais Rousseau n'en dit guère plus: le problème des «instruments propres à diriger l'opinion publique » est « une autre question qu' il serait superflu de résoudre pour vous (d'Alembert), et que ce n'est pas le lieu de résoudre pour la multitude ${ }^{45}$. Si cet art est superflu pour d'Alembert, on doit supposer que celui-ci le possède et que Rousseau ironise à son endroit. Quant à la multitude, il ne lui (c'est-à-dire nous) reste qu'à interpréter les indices de la Lettre ou à chercher ailleurs chez Rousseau. Regardons d'abord ce qu'il semble opposer explicitement ici au théâtre (la fête, le bal) à partir du bref exemple (du duel) auquel il renvoie la «multitude» dans ces pages.

42. Il est possible, selon Rousseau, d'agir sur les moeurs par les lois, le plaisir et l'opinion (Lettre, p. 74), et le théâtre n'y arrive sur aucun des trois fronts.

43. Lettre, PP. 143-154.

44. «Ni la raison, ni la vertu, ni les lois ne vaincront l'opinion publique, tant qu'on ne trouvera pas l'art de la changer $"$ : Lettre, p. 148.

45. Lettre, p. 144. 


\section{La fête}

En s'opposant au théâtre, Rousseau cherche à combattre cette "culture extérieure» qui en fait l'essence, à ses yeux, puisque l'admiration du public pour certains personnages et le ridicule d'autres, ne suscitent que des réactions passagères sans lien véritable avec la vie : le public reste au fond passif et séparé de l'action, tout en gouvernant en même temps ce qui se passe sur scène par l'inertie de l'opinion. Cette critique tout comme l'alternative que va esquisser Rousseau et ses difficultés, anticipent de nombreux débats sur le théâtre au $\mathrm{XX}^{\mathrm{e}}$ siècle ${ }^{46}$ : la mise en cause de son caractère de pur divertissement, de la réceptivité du public, de la séparation de celui-ci d'avec l'auteur et les acteurs, et de cette séparation entre les spectateurs que provoque leur rassemblement au théâtre alors que celui-ci prétend (et paraît) les réunir ${ }^{47}$. C'est bien une telle perspective anti-théâtrale de subversion des structures de la représentation en faveur d'un «événement» où auteur, acteurs et spectateurs ne feraient qu'un, qu'incarne la fête dans la Lettre ${ }^{48}$, et en particulier le fameux souvenir de celle de SaintGervais ${ }^{49}$. Rousseau célèbre une spontanéité qui rompt avec le paraître du théâtre : celui de la scène, mais aussi celui du public y venant davantage pour être vu que pour voir. Dans la participation improvisée de chacun dans la fête, par contre, il n'y a plus d'objet à voir, de signes à reconnaître, et l'on retrouve les rapports des hommes avant leur «chute » dans les comparaisons et les préférences individuelles ${ }^{50}$. La fête n'est visible par personne, et les femmes en particulier y perdent ce pouvoir de paraître et de se transformer qui les rapprochait du jeu des comédiens, et faisait des comédiennes, pour Rousseau, les plus dangereux ferments de «décadence » ${ }^{51}$.

46. Voir, par exemple, sur ce rapport, H. BÉHAR, Le paradoxe sur le théatre, dans Études françaises, $\mathrm{n}^{\circ} 8,1972$.

47. Lettre, p. 66. C'est un point dont d'Alembert n'aperçoit nullement la portée dans sa réponse: $o p$. cit., pp. 401-2.

48. Lettre, p. 223 et ss.

49. Lettre, pp. 248-9, note.

50. Le Deuxième Discours établissait cette ambivalence du développement des sentiments entre les individus; et RousSEAU écrit ailleurs : "Avec l'amour et l'amitié naissent les dissensions, l'inimitié, la haine. Du sein de tant de passions diverses, je vois l'opinion s'élever un trône inébranlable, et les stupides mortels asservis à son empire ne fonder leur propre existence que sur les jugements d'autrui. » : Emile, Pl. IV, p. 494.

51. Lettre, p. 212. 
L'art comme représentation semble donc aboli; mais ne serait-ce pas un art politique qui s'y est substitué ? L'évocation des plaisirs de la fête, en effet, fait rapidement place à la description soigneuse d'un événement qui laisse bien peu de place à l'improvisation et est doté d'une fonction sociale précise. Les bals de Genève $^{52}$ sont conçus, par Rousseau, pour la rencontre et le mariage des jeunes couples, l'éducation au respect des plus âgés, l'apprentissage des sentiments les plus sains - à l'image des fameuses fêtes des vendanges de Clarens, liées au travail productif et structurées avec précision par Wolmar ${ }^{53}$. "Donnez-lui (le peuple) des fêtes, écrit Rousseau, offrez-lui des amusements qui lui fassent aimer son état et l'empêchent d'en envier un plus doux. Des jours ainsi perdus feront mieux valoir tous les autres. Présidez à ses plaisirs pour les rendre honnêtes; c'est le vrai moyen d'animer ses travaux " 54 . Imbriquées d'emblée à des considérations de stratégie politique, les fêtes acquièrent ainsi une signification toute différente de celle qu'elles avaient au premier abord comme critique des médiations de la théâtralité. Tous les signes reviennent démultipliés mais recouverts de l'illusion de la spontanéité. Sans doute, le spectacle théâtral est aboli, mais si l'auteur n'est plus affiché, il n'en est pas moins présent et joue un rôle d'autant plus considérable qu'il est dissimulé. La question de l'organisation de la fusion festive (acteurs, auteur, spectateurs ; ou, si l'on veut, éducateurs et éduqués) reste entière en ce qu'elle fonctionne à présent souterrainement, clandestinement. La fête requiert encore son «artiste " pour en diriger les opérations. Il agit sur les comportements sans loi, sans contrainte, mais en utilisant justement un jeu d'apparences qui modèle à leur insu les participants. On le voit dans le paradoxe de la transparence qui préside à ces bals imaginés par Rousseau: ce ne sont que les interdits de l'Église, dit-il, qui créent comme par défi le goût des tête à tête entre amoureux, et ce besoin d'intimité n'est pas naturel; les fêtes de Rousseau ne lui font donc aucune place; mais ce caractère farouchement public, baigné de lumière, qu'il prône pour les bals et qui en garantit l'innocence, en transforme insensiblement le sens, en en faisant de nouveaux spectacles, des exercices d'un paraître sans limite, où les

52. Lettre, p. 238 et ss.

53. Voir N. H., se partie, VII.

54. Lettre, pp. 234-5, note. 
futurs époux deviennent malgré eux les acteurs d'une pièce conçue et dirigée, non par leurs parents - eux-mêmes acteurs -, mais pas un occulte metteur en scène 55 . Ainsi, en redoublant politiquement la spontanéité, Rousseau fait de la fête une manifestation particulièrement «spectaculaire» de l'art d'agir sur les hommes par le dedans.

Dans les belles pages que Starobinski consacre à ce thème ${ }^{56}$, il voit dans la fête la "forme lyrique de la volonté générale », en ce qu'elle symbolise l'abolition ultime de l'altérité et actualise ce que vise le Contrat social, c'est-à-dire la communauté affective, l'aliénation du moi de chacun dans un moi commun sans contrainte sociale ou psychologique. Dans ce rite d'unanimité où sont jouées les grandes valeurs de la Cité idéale, où s'identifient l'ordre et la spontanéité, se réalise ainsi cette fusion de l'art et de la vie qui structurait la critique des spectacles par Rousseau. Mais celui-ci ne cache nullement le caractère éphémère de cette fusion, et dans la Lettre c'est seulement comme référence critique, forme pure d'une transparence espérée, qu'est évoqué le « piquet » autour duquel nait spontanément la fête de Saint-Gervais, puisque la fête, pour Rousseau, c'est aussi (ou surtout) le bal. Il n'y a donc pas à opposer une logique répressive du contrat social au nom de la vertu, à la logique de jouissance sans contrainte qui serait celle de la fête, comme si le contrat assumait par ses structures les exigences de rapports à autrui que la fête par contre abolirait. La fête est d'emblée récupérée politiquement par Rousseau, et même lorsqu'elle est une évocation poétique, comme pour Saint-Gervais, elle reste profondément codée et pacifique. Elle n'est pas le lieu d'expression symbolique de pulsions asociales ou de transgressions, et elle ne met pas en cause les rangs sociaux mais les affermit au contraire. Elle exprime l'ordre social vertueux auquel les hommes aspirent naturellement mais qu'ils échouent à réaliser, et qu'il faut alors les inciter à forger au moyen de l'image que peut en donner la fête. Celle-ci fait donc partie intégrante du projet politique rousseauiste,

55. Rappelons à ce sujet le fameux épisode des oublies, des pains d'épice et des pommes, où Rousseau se fait l'opérateur d'une joyeuse spontanéité dont il peut alors jouir : Rêveries, Neuvième promenade, PI. I, Pp. 1090-3.

56. La Transparence et l'obstacle, Gallimard, Coll. Tel, 1971, pp. 116-121; voir aussi le beau livre de P. M. Vernes, La ville, la fête, la démocratie. Rousseau et les illusions de la communauté, Payot, 1978. 
mais elle y joue un rôle dont il faut souligner l'ambivalence et que la Lettre met en évidence : la fête y est à la fois une référence critique pour les hommes qui peut s'inscrire périodiquement comme rite dans la communauté; et elle est le terrain d'une manipulation des esprits, d'une action sociale calculée. Il y a donc une fête utopique légitimée par l'horizon de transparence qu'elle incarne éphémèrement au présent, et qui révèle en négatif les ravages du paraître; et une fête comme moyen politique de façonner cette transparence, qui fait sienne ces méfaits du paraître. Tantôt Rousseau l'évoque comme il évoque l'état de nature ${ }^{57}$, tantôt son sens est de véhiculer toutes les médiations nécessaires pour réinventer à l'état social les valeurs naturelles. Tout l'art de Rousseau est d'enrouler les deux aspects du phénomène l'un dans l'autre, ce qui ne va pas sans difficulté, on le comprend, puisque l'un désigne une pratique de la duplicité que l'autre récuse par principe ${ }^{58}$. Mais n'est-ce pas justement dans ce lien, dans ce glissement de sens, que réside la possibilité d'agir sur les coeurs?

C'est précisément ce que laisse entendre, très allusivement, l'exemple du duel dans la Lettre ${ }^{59}$, en faisant à sa manière la transition entre les spectacles inutiles et les spectacles vertueux. Rousseau y évoque l'échec des gouvernements à s'opposer à la pratique du duel, et considère que si l'on a échoué, c'est que l'on a «heurté de front l'opinion publique ». En disant préférer le nom de «cour d'honneur» à celui de ce «Tribunal des Maréchaux de France » institué pour réprimer le duel, il livre le fond de sa pensée sur cet échec, qui a été de n'avoir pas su prendre en compte l'opinion et su agir sur elle par les apparences. La force n'a aucun pouvoir sur les esprits et l'opinion n'est pas soumise à la contrainte, car la véritable autorité s'exerce «à l'intérieur de l'homme» et toutes les punitions, par exemple, ne font jamais que témoigner de

57. Ibid., pp. 129-130; et pp. 78-9 où Vernes renvoie à ce sujet au Ch. IX de l'Essai sur l'origine des langues: dans sa description de la naissance des liens sociaux, Rousseau évoque les " premières fêtes » nées spontanément par la rencontre des sexes autour des points d'eau (éd. Aubier Montaigne, 1974, pp. 136-7).

58. D'où le problème de la duplicité dans les textes mêmes de RousSEAU, et de ce type de déclaration, par exemple, adressée à la «multitude»: «Je puis me tromper mais non pas vous tromper volontairement. Craignez mes erreurs et non ma mauvaise foi », écrit-il dans la Lettre, p. 242, note. Dans le même esprit, voir la Lettre à C. de Beaumont, Pl. IV, pp. 966-7.

59. Lettre, p. 144 et ss. 
l'échec des gouvernements à agir sur cette opinion ${ }^{60}$. D'où l'importance des symboles du pouvoir et, dans ce cadre, des fêtes : non plus comme abolition des signes et de la théâtralité, mais au contraire par l'utilisation politique qu'elles permettent de faire des signes ${ }^{61}$. On est donc encore en plein spectacle, puisque la " cour d'honneur » est une sorte de scène où sera représenté le différend, permettant par la cérémonie de résorber la violence du duel : le conflit devient spectacle pour les adversaires eux-mêmes et pour un public dont ils se sentent tributaires, et cette transformation symbolique en désamorce les conséquences violentes. Cette représentation particulière suspend par l'imaginaire l'urgence pratique, la déplace sur le terrain d'une certaine illusion, d'un paraître assumé c'est-à-dire subordonné à la raison, mais sans que les protagonistes soient au fait de l'opération, ne réagissant qu'à l'image qu'elle leur renvoie d'eux-mêmes.

Ces quelques thèmes de la Lettre mettent ainsi en évidence la forme d'un problème décisif dans l'œuvre de Rousseau, mais auquel il n'apporte ici qu'une réponse "négative» en termes de résistance au développement de la corruption. L'action de ses trois grands « réformateurs» (le Législateur, le gouverneur d’Émile, Wolmar) est une tentative d'y répondre positivement.

\section{La ruse}

Le rôle du Législateur est introduit par Rousseau dans le cadre de la métamorphose de l'homme naturel en citoyen, dont l'acte du contrat constitue le premier moment fondateur. Rappelons le sens de son action sur les cœurs des hommes. Dans le Contrat social, seule la volonté générale est souveraine, mais la rectitude du peuple assemblé reste formelle car celui-ci « ne voit pas toujours » concrètement le bien qu'il recherche ${ }^{62}$, et le Législateur propose alors les lois particulières qui correspondent à la situation du peuple concerné. Situé dans le texte de Rousseau entre les chapitres

60. Voir Discours sur l'économie politique, Pl. III, pp. 249-50.

61. On sait que Rousseau insistera longuement sur l'importance des fêtes patriotiques, des cérémonies et des défilés qui, par leur discipline et leur représentation rigoureuse des distinctions sociales, favorisent le sentiment d'appartenance: Considérations sur le gouvernement de Pologne, PI. III, pp. 962-6.

62. Du Contrat social (C.S.), PI. III, p. 380. 
sur la loi et le peuple ${ }^{63}$, on voit où s'inscrit sa tâche : au niveau des mœurs, là où s'articulent les exigences de la raison et les passions des hommes. Cette action n'a pas seulement pour objectif de faire voir ce que le peuple ne voit pas, mais surtout de l'y mener à partir de ses ignorances et de ses coutumes, car la "vraie constitution" d'un peuple est toujours dans les cœurs. L'objectif est l'harmonie des mœurs et des lois, les lois ne pouvant agir sur les mœurs que dans la mesure où elles s'en nourrissent. Si cette action réussit, le peuple va se reconnaître dans les mesures élaborées par le Législateur, et celles-ci lui sembleront naturelles même s'il ne partage pas la science qui y aura présidé. Les lois transformeront cette nature dans la mesure même où elles sembleront y correspondre et l'exprimer. Ainsi, à l'occasion d'une consultation électorale, par exemple, le peuple «sanctionne de son vote les interprétations du Législateur » et « reconnaît comme son projet et sa nature une pure construction factice ${ }^{64}$.

On comprend alors que cette action ne puisse se contenter du langage de la raison. Si le peuple a besoin d'un tel guide, c'est bien que la voix de la raison ne lui est accessible qu'indirectement, si lui sont montrés non seulement «les objets tels qu'ils sont, (mais) quelquefois tels qu'ils doivent lui paraître ${ }^{65}$. Ne pouvant convaincre un peuple non encore institué, le Législateur doit avoir recours à la ruse, à un entraînement par les sentiments, à un maniement de l'illusion et de l'image afin de faire jouer en secret l'opinion. Parfait connaisseur des ressorts cachés de la nature humaine, il doit savoir se placer au-dessus de l'opinion pour gouverner les hommes par elle : «Je parle des mœurs, des coutumes et surtout de l'opinion; partie inconnue à nos politiques, mais de laquelle dépend le succès de toutes les autres : partie dont le grand Législateur s'occupe en secret, tandis qu'il paraît se borner à des règlements particuliers qui ne sont que le ceintre de la voûte, dont les mœurs, plus lentes à naître, forment enfin l'inébranlable Clef ${ }^{66}$. Il faut que les citoyens soient amenés à réaliser les fins rationnelles par leur propre liberté, en étant placés dans des situations telles qu'on

63. C. S., II, Ch. VII.

64. Comme le dit bien G. NAmER, Rousseau, sociologue de la connaissance, Klincksieck, 1978, pp. 317-8.

65. C. S., p. 380

66. C.S., p. 394 
leur donne, au niveau des motivations les plus profondes, des objectifs pour leur volonté sans qu'ils le sachent. D'où l'importance donnée par Rousseau à la religion, mise au service de la politique comme condition de la vie morale et de la vertu des citoyens: puisque le peuple requiert pour s'instituer la croyance en une autorité d'un autre ordre, le Législateur mettra ses pensées dans la bouche des dieux - et sa ruse sera légitime puisque les lois qu'il vise correspondent à l'ordre des choses voulu par Dieu et éclairé par la raison. Et pareillement pour les institutions et les cérémonies visant à former les âmes à l'oubli de soi et à la solidarité de l'État ${ }^{67}$.

Rousseau n'hésite pas à baptiser «libre » cette volonté manipulée mais droite, car en dehors de l'ordre de la cité, la liberté n'est pour lui que licence. Mais il faut souligner qu'ici le terme de liberté recouvre une ambiguïté, en désignant, d'un côté, les croyances illusoires des hommes utilisées par le Législateur pour agir sur eux en le leur laissant croire, dans la mesure où ils sont soumis à l'opinion, c'est-à-dire à l'image de liberté que leur renvoient les autres; de l'autre côté, c'est la valeur d'une conduite soumise à la loi du Législateur en tant que celle-ci incarne, à travers la ruse qui l'impose, la raison. Par cette liberté-soumission, Rousseau établit non un simple décalage entre l'entendement du peuple et celui du Législateur, mais une différence de nature que rien ne peut venir combler : le peuple reste, par principe, un enfant incapable d'user rationnellement de sa liberté. Celle-ci n'est qu'un instrument au service d'une liberté véritable dont il peut espérer jouir mais, paradoxalement, sans en être l'agent.

C'est avant tout sur cette question fondamentale qu'il faut distinguer la ruse du Législateur de celle du gouverneur d'Émile, car si celui-ci utilise tout autant une technique du paraître et s'il truque ses rapports avec son élève, il le fait dans la perspective de lui permettre de conquérir son indépendance, de se défaire de toute soumission. Rousseau souligne bien qu'il s'agit ici de l'éducation d'un homme non d'un citoyen. On pourrait dire qu'on le prépare à être lui-même Législateur, c'est-à-dire à se défaire du règne de l'opinion pour gouverner à son tour les autres hommes. Émile n'est pas l'un de ces êtres corrompus, ces «adultes-enfants», que

67. Sur les principes de l'éducation publique, voir Discours sur l'économie politique, Pl. III, pP. 259-262. 
prend en charge l'organisation sociale du Contrat : il est pleinement enfant, c'est-à-dire qu'en ce qui le concerne il n'est pas trop tard pour en faire un être vertueux si l'on sait l'accompagner dans son développement. On ne cherchera donc pas le levier de l'opinion pour agir sur lui, mais au contraire à le protéger de l'opinion, en limitant, par exemple, son vocabulaire et ses lectures ${ }^{68}$. Ce qui ne signifie pas qu'on cherchera à le raisonner ou à lui prêcher la bonne conduite, mais à le former à consentir à la nécessité de la nature pour lui apprendre ses besoins et ses limites. On préparera sa raison par la méthode des sens, la démonstration par les choses ${ }^{69}$. Mais cet apprentissage requiert qu'on le rende strictement obéissant à son gouverneur. La parole du maître doit avoir un caractère sacré pour Émile, le faisant s'incliner non devant un homme mais devant la majesté d'une loi dont il pressent la justesse sans en comprendre la raison, car c'est cette dépendance qui permettra de développer en lui des conduites raisonnables sous forme de réflexes. Ceux-ci seront inculqués en truquant la nature ${ }^{70}$, afin qu'Émile ne fasse que ce qu'il veut mais ne veuille que ce que le gouverneur décide qu'il fasse. Émile apprend à son insu ce qu'est sa liberté dans la mesure même où ce joug lui est imposé et où il en fait l'épreuve par sa propre volonté.

Si la finalité de cette action sur l'enfant est différente de celle du Législateur, sa structure est la même, puisque c'est dans la mesure où ce joug imposé est caché qu'il peut être librement consenti ${ }^{71}$. L'obéissance à la nécessité n'étant pas discernable par la raison chez l'enfant ( $\mathrm{ni}$, nous l'avons vu, chez la plupart des adultes), la liberté se confond avec le réflexe induit, jusqu'à ce qu'on puisse s'adresser à cette raison. À l'adolescence d'Émile les modalités d'apprentissage changent, confortant en lui l'illusion de la liberté pour contenir sa révolte; mais l'approche «simple et vraie » du gouverneur et son appel à la sincérité de l'élève ne sont que les masques qui conviennent à ce moment de développement.

68. Émile, Pl. IV, pP. 454-6 et p. 298. Il est vrai que le gouverneur agira sur lui en utilisant son amour-propre, et c'est là un travail délicat et même dangereux car on risque justement de le soumettre ainsi à l'opinion (p. 536).

69. Il faut se garder avant tout de s'adresser trop vite à la raison, comme le fait Locke, Émile, pp. 317-320.

70. Par la ruse du «canard aimanté», par exemple: Émile, pp. 437-440.

71. L'enfant doit être libre et docile, selon Julie, dont la pédagogie retrouve celle de l'Émile: N. H., se partie, III. 
Et ainsi de suite, jusqu'à une échéance qualitative qui distingue Émile des citoyens du Contrat, puisqu'il peut espérer rejoindre la lucidité de ces «hommes-dieux» que sont son précepteur, le Législateur et Rousseau lui-même, en échappant radicalement à l'empire de l'opinion ${ }^{72}$.

Un tel objectif dépend du diagnostic porté sur la situation moral des hommes auxquels on a affaire, tout comme l'état de la société genevoise décidait, dans la Lettre, de la teneur de l'attaque de Rousseau contre les spectacles. La duplicité est nécessaire dans tous les cas, mais selon des modalités et des finalités très différentes, car dans un cas c'est pour jouir des effets de cette duplicité sans espoir d'enlever les masques, dans l'autre c'est pour atteindre la conscience de sa nécessité afin de l'utiliser à son tour ${ }^{73}$. On voit alors que les différences de diagnostic quant au degré de dénaturation et donc au dosage de duplicité, recouvrent une distinction plus fondamentale entre l'individu et le peuple dans leur rapport à l'opinion. Ce qui manque aux peuples c'est la possibilité même de la maîtrise de leurs passions telle que peut la développer l'individu à partir de ses virtualités. Les peuples sont et restent "enfants ", même si ces «enfances » peuvent être plus ou moins pures, c'est-àdire réformables (Genève, Pologne, Corse); et donc la politique de Rousseau fige les principes de duplicité de sa pédagogie de l'enfant, non dans la perspective du devenir adulte, mais dans celle de gérer raisonnablement le goût des images et la dépendance inhérents aux peuples. Certes, on a souvent fait l'hypothèse que dans l'économie générale de l'œuvre de Rousseau, l'éducation de l'homme et celle du citoyen doivent être comprises comme complémentaires, les institutions politiques du Contrat et la maturité individuelle d'Émile étant requises l'une par l'autre pour constituer une éthique sociale cohérente. Mais l'articulation explicite des deux composantes ne se trouve pas chez Rousseau, qui met d'ailleurs en garde contre toute tentative de réaliser en même

72. Émile, dit Rousseau, est "placé derrière le théâtre, voyant les acteurs prendre et poser leurs habits, et comptant les cordes et les poulies dont le grossier prestige abuse les yeux des spectateurs»: Émile, p. 532. On peut se demander toutefois si Émile se montre à la hauteur de sa vocation dans les épreuves que lui fait subir Rousseau après l'achèvernent de sa formation; voir Émile et Sophie ou les Solitaires, Pl. IV

73. Dans le cas des spectacles, il ne s'agit, plus modestement, que de résister à la corruption des mœurs à Genève et de modérer les effets de celle-ci à Paris. 
temps l'éducation publique du citoyen et l'éducation particulière de l'homme. Sa pensée sociale et politique ne ménage donc pas la possibilité d'une mobilité intellectuelle et morale telle que l'Émile en dessine les conditions, et l'on doit conclure qu'une telle perspective n'a de sens que réservée à des individus d'exception, d'heureux élus chargés de manier la ruse pour agir sur l'opinion, en ayant pris conscience présisément du défaut rédhibitoire qui afflige l'existence collective. C'est à certains individus que revient la tâche de pallier la carence du peuple.

\section{L'échec}

Plutôt que d'examiner de front cette hypothèse, nous nous contenterons d'évoquer pour finir le troisième « réformateur »Wolmar dans La Nouvelle Héloïse -, qui est davantage un individu singulier qu'une instance dans une théorie politique ou pédagogique, et dont l'action et la ruse débouchent sur des résultats beaucoup plus incertains. Cet exemple nous paraît fondamental, car si l'autorité de Wolmar à Clarens ruine plus que jamais l'image d'un Rousseau seulement sincère et naif, elle interdit tout autant d'en faire seulement le champion de la duplicité et de la raison calculatrice.

C'est à l'échelle de l'économie domestique plutôt que politique que Rousseau fait les descriptions les plus graphiques de cette stratégie du paraître ${ }^{74}$ (le Contrat social, en particulier, restant très allusif à ce sujet). Le royaume de la transparence des cours à Clarens se révèle, en effet, être en même temps celui des apparences ; et ce règne de l'immédiateté de la vérité, être celui des médiations et de l'illusion ${ }^{75}$. L'occultation de l'autorité au nom de la liberté est ici encore érigée en système, puisque les rôles sont distribués une fois pour toutes, que les domestiques resteront ce qu'ils sont : des enfants, dont Rousseau dit bien en préface qu'ils « ne goûtent pas mieux la raison nue que les remèdes mal déguisés ${ }^{76}$. Il est vrai

\footnotetext{
74. N. H., $4^{\mathrm{e}}$ partie, X.

75. Dans ses très belles analyses sur La Nouvelle Héloüse (qui sont de loin ce qu'il y a de meilleur dans son ouvrage), Philonenko caractérise Clarens comme un rêve de transparence qui se transforme en cauchemar de surveillance : op. cit., Vol. II, L'espoir et l'existence.

76. N. H., $2^{e}$ préface, Pl. II, p. 17.
} 
que Wolmar se distingue du Législateur par l'étendue de son pouvoir : nul pour ce dernier, c'est-à-dire résidant seulement dans sa sagesse, celui de Wolmar est par contre absolu. Mais leur modèle d'autorité (le père de famille) et leurs principes d'action sur les esprits et les cœurs sont semblables : peu de lois ou de règles positives qui ne font que produire la tentation de les enfreindre, mais l'établissement discret d'usages, plus puissants car reposant sur l'opinion; et pour y arriver, l'utilisation systématique de l'illusion de la liberté, la fabrication soigneuse de motivations par l'observation et la manipulation des passions, l'objectif étant que les serviteurs de Clarens s'imaginent vouloir tout ce que Wolmar leur fait faire. Pour l'essentiel, celui-ci combine deux méthodes : il utilise l'esprit de concurrence, un système de primes, etc. (suscitant tous les sentiments si sévèrement condamnés dans le Deuxième Discours), afin de laisser croire au serviteur qu'il travaille pour luimême quand il travaille pour le bien commun; et en même temps Wolmar encourage la soumission à l'opinion pour que les serviteurs ne pensent à eux-mêmes que par rapport à la communauté entière et en particulier à travers l'approbation du maître et de la maîtresse de la maison. De cette manière la transparence à Clarens est soigneusement construite par la technique de la «main cachée » ${ }^{77}$; et elle semble se réaliser dans l'exacte mesure où elle est soustendue par l'illusion - ou plutôt, comme le suggère Philonenko, elle devient peut-être elle-même la grande illusion : « La transparence ne serait-elle pas le voile qui cache le grand secret ? ${ }^{78}$.

Mais cette main cachée ne se limite pas à l'organisation du travail à Clarens, puisqu'elle modèle aussi les rapports de conjugalité et d'amitié de Wolmar, en particulier avec Saint-Preux qui est susceptible d'une plus grande mobilité psychologique que n'en était accordée aux domestiques. Comme les hommes corrompus du Deuxième Discours, celui-ci reste déchiré entre ses impulsions naturelles (amoureuses) et l'ordre social qu'incarne Clarens. Il s'agit donc de l'intégrer à la communauté de son ancienne amante, de le faire rompre avec un passé qui le fascine, pour lui faire atteindre, s'il en est capable, ce qu'il appelle lui-même «la vie

77. Le modèle de cet art est la transparence artificielle du jardin de Julie, l'Élysée, où le développement «spontané » de la nature est le résultat d'un travail précis sur une longue période : N. H., 4e partie, XI.

78. Philonenko, Vol. II, op. cit., p. 196. 
morale " ${ }^{79}$. Pour cela, Wolmar observe Saint-Preux comme il le fait avec ses enfants, lui fait subir des épreuves pour sonder son cœur, lui fait sentir sa totale confiance afin de mieux gagner celle du jeune homme, et surtout fait jouer l'arme clef de l'opinion, car cette «âme faible » a le goût de l'estime des autres, avant tout celle de Wolmar lui-même ${ }^{80}$. Bien différemment, mais avec la même duplicité, Wolmar agit sur Julie et son propre passé pour faire naître en elle la transformation de son amour pour Saint-Preux en simple tendresse. Il incite les anciens amants à vivre ouvertement leur affection pour en désamorcer la force, les ramène sur les lieux de leur idylle pour les désacraliser, consent à ce qu'ils s'embrassent devant lui ; bref, tout est fait pour qu'ils génèrent eux-mêmes leur renoncement ${ }^{81}$. Et Wolmar n'hésite pas à gagner la complicité de Claire, meilleure amie de Julie, pour la mise en scène requise, justifiant la tromperie par la fin vertueuse recherchée ${ }^{82}$. On peut dire qu'ici encore Wolmar ne vise rien d'autre que la liberté des anciens amants, mais c'est une liberté qui n'est atteignable qu’à leur insu — du moins en partie - et au moyen d'une manipulation de leur liberté naturelle.

Cela dit, la force de La Nowvelle Hélö̈se est justement que Rousseau ne s'en tient pas à une simple opposition de la froide raison et du désordre des passions ; et l'épaisseur du problème est mise en évidence de manière dramatique par l'ultime composante des rapports de Wolmar à Julie et à Saint-Preux, et qu'il faut bien désigner comme l'échec de toute son entreprise. On ne peut nier le succès de son action sur les domestiques à Clarens, et, à travers Julie, sur ses enfants. Mais avec des individus adultes et sophistiqués, les principes pédagogiques de l'Émile sont inutiles car on a affaire à des êtres déjà corrompus, et les manœuvres conçues pour gouverner le peuple sont inappropriées puisqu'on s'adresse à de "grandes âmes" sinon à des êtres d'exception. Ce n'est pas au niveau de ses rôles politique, économique ou paternel qu'échoue donc Wolmar, mais au niveau plus intime de son existence affective et spirituelle, sur un terrain qui semble échapper aux duplicités politique ou pédagogique vues plus haut : à ce niveau, le

79. N. H., $5^{\mathrm{e}}$ partie, VIII, p. 611.

80. Ibid.; voir aussi sa lettre sur l'athéisme de Wolmar : $5^{e}$ partie, $V$.

81. N. H., $4^{\mathrm{e}}$ partie, VII et XII.

82. N. H., $4^{\mathrm{e}}$ partie, XIV. 
modèle de l'action détachée du Législateur ou du gouveneur se révèle beaucoup plus fragile, car cette action met directement en jeu le statut psychologique de Wolmar.

Celui-ci peut être précisé en rapprochant sa duplicité de la duplicité amoureuse de Julie et Saint-Preux lorsque se noue leur idylle au début du roman ${ }^{83}$. Le jeu de séduction mené dans les premières lettres au nom de la vertu, révèle d'emblée l'enjeu éthique central de La Nouvelle Héloïse : le problème de la duplicité envers soi, de la capacité des hommes de se mentir, de se duper eux-mêmes. C'est essentiellement cela qui distingue Wolmar, car face à tous ces personnages qui se dupent, il est celui qui dupe tous les autres ${ }^{84}$. Il connaît les passions des hommes mais ne les vit pas lui-même, ne cherche pas à se mentir, n'a pas ce goût de l'illusion qui structure l'existence de ceux qui l'entourent. Mais ne se mentant pas à lui-même, Wolmar doit leur mentir. Comment se rapporter à eux sinon par leurs chimères, par leur imagination, par leur soumission à l'opinion? Qu'est-ce que l'amour chez Julie, chez Saint-Preux, d'ailleurs, sinon ce goût de tout miser sur l'estime d'un autre, de s'y aliéner radicalement? Wolmar se rapporte à lui-même sans la médiation de l'image et sans religion, ce qui le rend étranger aux hommes, mais c'est ce qui en fait aussi un formidable fabriquant d'images. On retrouve à travers lui toute la problématique de la théâtralité, car c'est un homme de spectacles, mais qui semble échapper à toutes les illusions, et dont la virtuosité est sans défaillance car il n'a pas à dissimuler des passions qu'il n'éprouve pas. Mais n'ayant besoin d'aucun masque et ne se prenant pas au jeu de l'acteur, il est pourtant par son action un être de masques, il n'est même que masques. S'il n'est pas pris dans la tromperie théâtrale, c'est qu'il en est le metteur en scène; mais chacun sait qu'au théâtre il n'y a pas plus comédien que ce grand solitaire, sauf que sa scène est toujours ailleurs, car son public c'est toute la représentation: acteurs, public et postérité compris. Son illusion est de croire qu'il échappe à tous les rôles, qu'il n'a pas à en choisir un, mais il ne peut y échapper. Pour Wolmar, c'est le rôle

83. Voir àce sujet, L. CROCKER, «Julie ou la nouvelle duplicité », dans Annales de la Société J. J. Rousseau, $\mathrm{n}^{\circ} 36,1963-5$. On peut regretter toutefois le ton moralisateur de ce texte qui semble négliger la portée philosophique de la duplicité chez Rousseau, en lui opposant implicitement une éthique naïve de la coïncidence avec soi.

84. Ibid., p. 146. 
du sage, avec le masque de la raison, le masque de Dieu, et c'est ce masque qui menace de tomber à la fin du roman. Face à Julie et Saint-Preux d'abord, car il échoue dans sa stratégie conjugale: leurs sentiments restent indomptés ${ }^{85}$; Wolmar ne réussit pas à briser leur attachement aux chimères de leur passé, à leur faire perdre le goût de l'ombre, de l'imaginaire, pour leur faire embrasser les exigences de l'ordre et de la coïncidence avec soi. Mais Wolmar échoue aussi pour lui-même à la mort de Julie, lorsque l'insuffisance de son athéisme se révèle par sa solitude et son déracinement. Ironie de ce faux acteur qui n'allait au temple que " pour les enfants et les domestiques », il semble bien qu'il rejoigne le monde des hommes par la grande porte de la religion en découvrant sa foi ${ }^{86}$. Wolmar semble près de se tourner vers le monde des chimères exalté par Julie, en y voyant le moyen de sortir de sa prison rationnelle ${ }^{87}$.

La folle duplicité des hommes appelle la duplicité rationnelle de Wolmar ; leur mensonge à soi, son mensonge aux autres ; leur théâtralité, ses mises en scène. Mais si son action retrouve bien la critique des acteurs de la Lettre qui, comme tous les hommes corrompus, sont esclaves du paraître, son échec suggère que les metteurs en scène n'échappent peut-être pas à la duperie. Wolmar n'est certes pas un de ces «Philosophes » en quête de public que Rousseau rapprochait des acteurs; il est un sage, mais il n'en est pas moins homme (comme l'est le Législateur ou l'éducateur), et la victoire de la raison, dont relève toute la logique de La Nouvelle Héloïse, n'est pas univoque: elle reste en suspens, renvoyée au jugement et à la sensibilité du lecteur. Dès lors, entre une Julie trop apte à se duper elle-même, s'étant façonnée grâce à Wolmar une vaste illusion qui l'a tuée, et un Wolmar trop apte à duper les autres et se retrouvant seul, c'est Saint-Preux qui apparaît comme une sorte de héros philosophique sans victoire. Par rapport à la conversion vertueuse de Julie et à l'incarnation de la raison de Wolmar, il reste sans doute en deçà de l'une et de l'autre par sa faiblesse, mais l'une et l'autre révèlent néanmoins leurs limites.

85. N. H., $4^{\mathrm{e}}$ partie, XVII ; et la fin de la dernière lettre de Julie : $6^{\mathrm{e}}$ partie, XII.

86. N. H., Ge partie, XII ; et la note de B. Guyon, Pl. II, Pp. 1811-2.

87. Mais trop tard. Peu importe, dira-t-on: Wolmar n'était pas vraiment hormme et les dieux ne sauraient nous émouvoir ; le drame humain est celui de Julie et de SaintPreux. Mais Rousseau ne s'est-il pas aussi peint, pour une part, en Wolmar? 
Saint-Preux semble témoigner alors de ce douloureux paradoxe, que l'homme se dupe, par son imagination, par les dédoublements qu'il ne cesse d'opérer en lui-même, par une foncière théâtralité qui le mine ; mais que cette duperie est constitutive de son être, que les images lui sont nécessaires et forment l'étoffe même de son existence. L'échec de Wolmar, qui est seulement suggéré au lecteur comme un sens possible qu'il peut choisir de poursuivre en refermant le roman, en serait comme l'indication, comme la difficile reconnaissance. Par cette suggestion, Rousseau semble refuser de se satisfaire d'une éthique des hommes supérieurs incarnant la raison et agissant sur l'opinion dans la mesure où ils y échappent radicalement eux-mêmes; un simple "réalisme » de la ruse et de la duplicité ne saurait suffire. Mais si une telle éthique est ici problématisée, cela ne signifie nullement que toute stratégie du paraître se trouve par là dénoncée, au profit d'un idéalisme de la fin transparente accessible par la spontanéité de l'homme naturel. Rousseau, on l'a souvent souligné, ne se détourne pas de la raison au nom du sentiment, il tente plutôt d'élaborer leur collaboration. On comprend alors que dans un roman où il investit tant de luimême, la mort de Julie doive recevoir une contrepartie par cette indication des limites existentielles de la lucidité.

Un tel dénouement ne peut que résonner à travers toute l'œuvre de Rousseau. Il fait écho aux problèmes posés et laissés en suspens dans la Lettre, car si le Contrat social et l'Émile prennent pleinement en charge son interrogation sur l'opinion et y répondent sur leur terrain respectif, La Nouvelle Héloüse semble la reprendre pour la laisser en suspens à nouveau. Ensuite, en ce qui concerne l'enjeu de la querelle religieuse genevoise entre l'exigence de lucidité critique et l'exigence d'un consentement aux apparences et à l'autorité, c'est cette dernière que rappelle l'échec de Wolmar en l'appronfondissant: non seulement il y a un domaine de vie symbolique qui est irréductible dans les rapports entre les hommes, exprimant les limites du savoir et de l'action rationnelle, - cela Wolmar plus qu'aucun autre le sait - ; mais si la politique et la philosophie doivent bien utiliser et comprendre les apparences et les images, on ne peut oublier qu'elles en sont aussi elles-mêmes investies. D'où, comme nous l'indiquions en commençant, la constante problématisation et le redoublement chez Rousseau de toute action réformatrice aussi sage soit-elle, par l'ambiguité de 
son rapport à l'opinion. Le cheminement de l'écrivain Rousseau n'en porte-t-il pas témoignage d'ailleurs, par la dimension autobiographique de la fin de son ouvre? Celle-ci peut être envisagée, en effet, comme l'aboutissement tragique du questionnement inauguré par le Premier Discours et la Lettre. Sans doute espèret-il encore par l'autobiographie combattre ou échapper à la prison du paraître, au mensonge de la théâtralité; mais non plus au moyen des «spectacles » de la fiction ou des jeux d'apparence de la politique. L'échec de l'action et de la ruse devient lui-même spectacle, car il ne s'agit plus de réformer la société ou de changer l'homme, mais de rendre compte de cet échec, et par là peut-être de le transformer en consentement ou en sérénité ${ }^{88}$. Si on ne se débarrasse pas du paraître de la vie en ce qu'elle est spectacle, on va parier sur le spectacle de la vie comme spectacle. L'autobiographie signale alors l'abandon de toute éthique sociale, de toute intention réformatrice, car il s'agit moins maintenant de guérir le mal par le mal que de montrer le drame des essais de guérison, de se montrer échouant mais de bonne foi. Par le spectacle de sa bonne foi, le réformateur déçu tente ainsi de devenir la mauvaise conscience de ses lecteurs et de la société entière ; mais par là il échappe moins que jamais, on le voit, aux tourniquets du paraître. Par delà la duplicité et la transparence, l'ultime leçon de Rousseau en est une d'irréconciliation, où moyen et fin se minent l'un l'autre au point ici de se confondre. *

Université LAVAL et Université de PARIS IV

88. Philonenko parle à ce sujer du « rétrécissement stratégique » et de la "stratégie de retraite " que dessine l'évolution du Contrat social à l'Émile aux Confessions à partir des enjeux mis en place dans La Nouvelle Héloise : à défaut de guérir le monde, le médecin-philosophe se résout à se guérir lui-même : op. cit., Vol. III, Apothéose $d u$ désespoir, p. 82 et 283.

* Ce texte s'inscrit dans un travail de recherche postoctorale rendu possible par une bourse du Conseil de recherches en sciences humaines du Canada. 